\title{
Interview with Dr Bernard Pécoul, Executive Director of the Drugs for Neglected Diseases Initiative
}

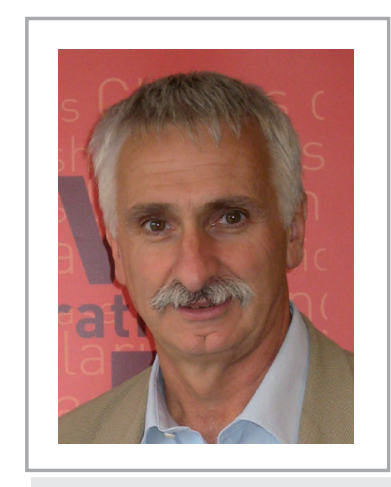

A significant number of product-development partnerships have arisen in the past 10 years to tackle diseases that mainly affect developing countries. With their coherent research and development leadership, they have become key players in identifying gaps and overcoming bottlenecks in order to deliver medicines to those who need them most in the developing world. Dr Bernard Pécoul is the Executive Director of one such partnership, the Drugs for Neglected Diseases Initiative (DNDi). Under his leadership, the DNDi has built the largest and most robust R\&D portfolio for three of the most neglected diseases. He speaks to Future Medicinal Chemistry about challenges facing neglected disease R\&D and the DNDi's ongoing work.

Future Medicinal Chemistry spoke with Dr Bernard Pécoul in February 2009.

Q The R\&D landscape has evolved greatly over the past $10-15$ years.

What influence are new initiatives, such as product development partnerships (e.g., the Drugs for Neglected Diseases Initiative) having on stimulating drug development?

I think the main influence has been to bring money to the table, but more than that, the influence has been to develop a needs-driven R\&D agenda. Initiatives such as the Drugs for Neglected Diseases Initiative (DNDi), and others in the field of malaria and TB have contributed to attracting partners in order to achieve certain objectives. This is very dynamic. What still needs to be done, however, is to create stability in the system, so as to develop something that can survive in a different intellectual property (IP) and regulatory environment as well as under sustainable sources of funding, thereby allowing this model to be translated into something long term.

Since its foundation in 2003 by five public sector institutions, Médecins sans Frontières \& the UNDP/World Bank/WHO's Special Programme for Research and Training in Tropical Diseases, how has DNDi grown?

We started from scratch and have been able to develop a portfolio of projects, some of which we have already delivered on. I think we have at least been able to transform the vision of our model into something that is fairly concrete, to attract partners around a set of objectives, as well as to carry out R\&D activity around the most neglected diseases. In disease-endemic countries, our founding partners, such as the Indian Council for Medical Research, the Kenya Medical Research Institute, the Oswaldo Cruz Foundation (Fiocruz) in Brazil and the Malaysian Ministry of Health, play a key role in helping us carry out our mission objectives.

Q The DNDi concerns itself with diseases limited to tropical countries. Which other organizations are doing significant work in this area?

The DNDi is not totally isolated, but we are perhaps the only organization whose objective is to develop new treatments exclusively for neglected diseases.

Some other initiatives contribute, but these have many other goals. The DNDi's specific aim is to concentrate the majority of its efforts on this group of diseases. We have plenty of partnerships with other organizations. I could mention, for example, the WHO Special Programme for Research and Training in Tropical Diseases, which has helped facilitate the creation of an environment for numerous projects as well as training. We are taking advantage of all these other initiatives.

\section{Bernard Pécoul}

Drugs for Neglected Diseases Initiative, 15 Chemin

Louis-Dunant, Geneva,

1202, Switzerland

Tel.: +4l 229069230

Fax: +4I 229069231

E-mail: dndi@dndi.org 


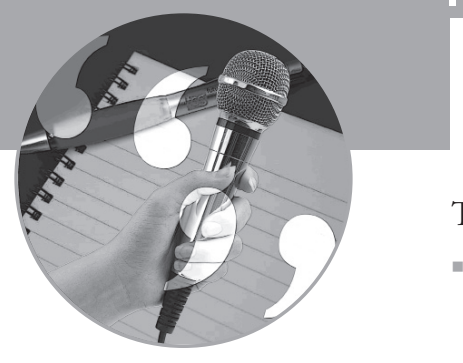

The DNDi's objectives are to:

- Deliver six to eight new treatments by 2014 for leishmaniasis, sleeping sickness, Chagas disease and malaria

- Establish a robust portfolio for the new generation of treatments

Can you sense change in the political attention given to your organization and others like it?

The change over the past 10 years. In 1999, we were describing a 'stand-still' in neglected

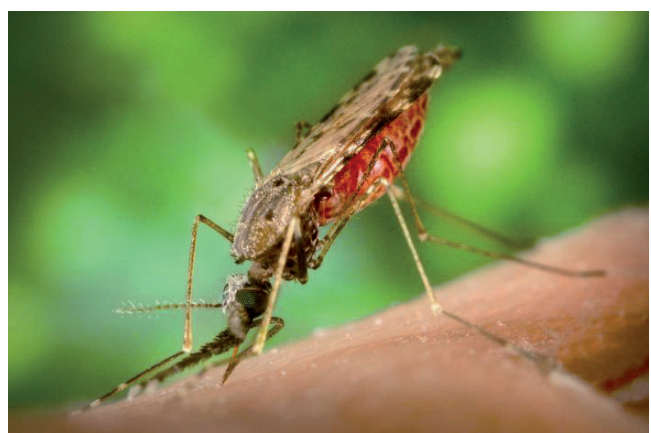

Malaria vector, the Anopheles albimanus mosquito. disease R\&D. It was a drastic situation given that very few projects had been developed over the past 25 years. I think that 10 years later there is now at least a consensus on future directions, with all partners (public and private) agreeing that many different models and initiatives could be part of the solution. Therefore, I think there has been a big change. We started from a very negative situation and now we are seeing the beginning of a response.

In terms of developing new partnerships \& consortia to promote and stimulate drug development, what do you find to be the main challenges?

There are many challenges. Since we are working in a very neglected field, classical incentives that have been in place for the past 30-40 years are not adapted to the situation of neglected diseases - in particular the IP system, but also the regulatory system, because in developing new drugs we are very dependent upon the US FDA and the EMEA. Therefore, we need to address these issues. In developing clinical trials, for example, we started from scratch and have begun to build what we might call 'clinical trial platforms and networks'. However, these still need to be consolidated into sustainable partnerships.

\section{Sleeping sickness}

Sleeping sickness, or human African trypanosomiasis, threatens 60 million people in subSaharan Africa. The WHO estimates 50,000-70,000 people are affected every year, resulting in major social and economic consequences. The Democratic Republic of the Congo alone accounts for two thirds of reported cases. The disease is transmitted to humans by tsetse flies, whose bite produces two stages of the disease: stage I often goes undiagnosed and stage 2 is fatal if left untreated.
Q Technology and information transfer are very much part of the culture of collaboration. How do you feel this philosophy is influencing the work being undertaken by the DNDi?

For the DNDi, technology transfer towards the South or to disease-endemic countries is one of the key objectives of our mission. We believe that the problem of neglected disease drug development will be solved if we develop the capacity to perform the majority of R\&D in the countries directly affected. Therefore, this is a way of ensuring a sustainable response and is very much, I agree, part of the culture we have been discussing.

The distinction between 'neglected diseases' and 'most neglected diseases' is an important one. One 'most neglected disease' is sleeping sickness (human African trypanosomiasis(HAT), for which 36 countries are considered endemic. At the end of 2008, Phase III clinical trials were successfully completed for nifurtimox-eflornithine combination therapy (a simplified treatment for stage 2, the advanced form of the disease) at three DNDi-supported sites. Can you update us on these results, their significance \& what the future holds for this treatment?

Since we concentrate our efforts on 'most neglected diseases', we started with a dramatic situation - for example, in the case of sleeping sickness most patients are still receiving highly toxic, arsenic-based drugs such as melarsoprol. This is why our strategy was to first improve the situation with existing drugs; the nifurtimox-eflornithine combination therapy (NECT) project was part of this. We developed this clinical trial in the Republic of the Congo and in the Democratic Republic of the Congo and have conducted the study. We hope that in next few months this new treatment will be recommended as a first-line treatment for sleeping sickness. In parallel, however, we also want to develop new treatments that are more adapted to the needs of the patients in the field. 


\section{NECT in a nutshell}

Compared with eflornithine monotherapy, NECT would reduce the number of infusions of eflornithine from 56 to 14, the treatment duration from 14 to 10 days and the number of infusions per day from four (every 6 h) to two (every $12 \mathrm{~h}$ ). In addition, resistance is also less likely to develop as the two drugs have different modes of action, thus mutual protection is to be expected.
In summary, the NECT Phase III pivotal study conclusively demonstrates that NECT is a safe, effective treatment for stage 2 HAT and is more practical than the currently existing treatment.

\section{Q What are the problems with current treatments?}

Existing therapies are often toxic, prohibitively expensive, and need to be administered for long periods by injection, therefore not adapted to remote settings. In the case of sleeping sickness, diagnostic tools are inadequate and the few drugs available are toxic (i.e., melarsoprol, which kills one in 20 patients), difficult to use and increasingly ineffective in preventing death (up to $60 \%$ resistance). For visceral leishmaniasis (VL; also known as black fever), major obstacles include invasive diagnostics, lenghty treatments (30 days), and drug resistance (up to 65\% in India). Meanwhile, as regards other diseases, no drugs exist at all to treat patients with Buruli ulcer or chronic Chagas disease.

\section{What efforts \& progress are being made by the DNDi in stimulating \&} advancing R\&D for other neglected diseases?

First of all, we have developed two fixed-dose combination therapies, known as ASAQ and ASMQ, as part of the FACT projects for the treatment of malaria, however, with our partners we are also starting to build a relatively strong portfolio for a wide range of other diseases, for which we hope to develop new therapies in the next few years.

So, the development of the co-formulations is a very concrete achievement, but our wider portfolio is key for further success.

\section{FACT projects: ASAQ \& ASMQ}

The multipartner fixed-dose, artesunate combination therapies (FACT) project, created in 2003 by Médecins Sans Frontières and then transferred to DNDi in coordination with WHO TDR in 2003, is a response to a specific need to control malaria resistance by producing coformulation therapies from existing antimalarials drugs.

- FACT has delivered the first two fixed-dose ACTs with paediatric strengths:

- ASAQ: fixed-dose combination of artesunate and amodiaquine for the treatment of malaria in sub-Saharan Africa launched in March 2007; registered in 24 diseaseendemic countries; produced in landmark partnership with Sanofi-Aventis; obtained WHO prequalification in October 2008; 5.4 million of treatments delivered in 2008; www.actwithasaq.org.

- ASMQ: FDC of artesunate and mefloquine for treatment of malaria in Latin America and Asia; registered in Brazil in March 2008; key partner: Farmanguinhos/Fiocruz; SouthSouth technology transfer underway to Cipla for availability in Asia and Africa; in use by Brazilian national authorities as part of ongoing intervention study $(25,000+$ patients); www.actwithasmq.org.

\section{Q How are you working to ensure that ASAQ \& ASMQ become accessible as} quickly as possible to patients?

We are already increasing distribution and use of ASAQ. The drug is included in many treatment guidelines and is being recommended at the national level in 24 African countries. So, we are already at the implementation stage. Along with our partners, we are also carrying out effectiveness studies and bringing on board support for the pharmacovigilance plan (safetyorientated studies to delineate a drug's risks, benefits and optimal use) to monitor the effects of the drug. The expectation for 2009 is to have more than 20 million effective treatments of ASAQ available for patients.

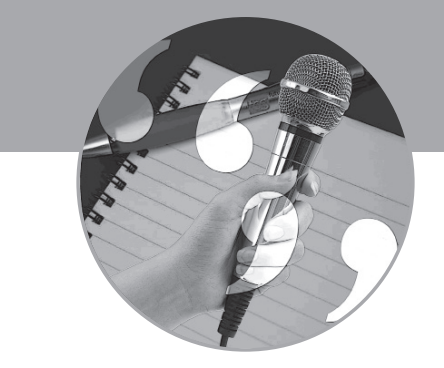

\section{Chagas disease}

Chagas disease (human American trypanosomiasis), infects approximately 8 million and puts 100 million at risk in Central and South America. It is transmitted to humans by kissing bugs. The acute form of the disease kills one in 20 (primarily children). The chronic infection leads to often fatal cardiac and intestinal complications. 


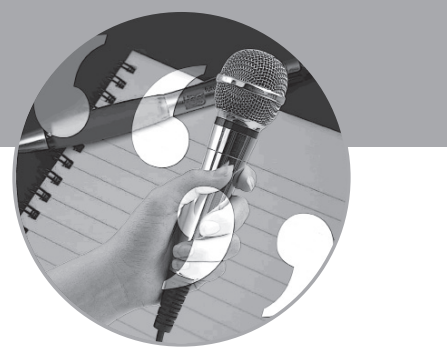

In terms of ASMQ, the product is already being used in Brazil, and we are in the process of extending its use to the rest of Latin America and then Asia. For the time being there is still a need to improve documentation for the use of this drug combination in Africa to gain early evidence of its effectiveness.

Can you provide us with a status update on your work concerning new treatments for visceral leishmaniasis \& Chagas disease?

We are making quite a lot of progress on visceral leishmaniasis (VL) and feel we should be able to introduce a drug combination in the next few years. By combining the treatment we could improve management of the patients as well as reduce the risk of resistance. If we use monotherapies, we are always faced with the possibility of resistance developing in a relatively short period of time. The disease is complicated in that it covers several continents, and we have documented that one regimen could be very active in one part of the world but less active elsewhere. We therefore need to develop various clinical substances in parallel before making recommendations.



Boy in the Democratic Republic of Congo, one of the countries in which the NECT clinical trials were carried out. majority of the 500,000 new cases each year occur in the rural areas of the Indian subcontinent, Brazil, Sudan and Ethiopia. It is estimated that only $30 \%$ of cases are reported.

We are less advanced in the case of Chagas because this is a more complex disease. We have spent 3 years considering the needs and complexities to be overcome in order to develop drugs. We expect in 2010 that we will have a better formulation for children. Currently, treatment is not adapted and so, for example, we hope to be able to offer tablets for children, who are the main victims of the acute form of the disease.

Q The DNDi has taken a bold stand with its IP policy, which resulted in production of ASAQ \& ASMQ without patent protection. Do you feel your stance on this issue has had an impact on influencing wider opinion?

Again, over the past 10 years, things have changed a lot. To be modest, I feel what we have brought to the table has to some extent contributed to changing wider opinion. The fact we have signed a partnership with a large company like Sanofi Aventis, for instance, and that they accepted nonexclusivity, is a breakthrough, and should, I believe, be used for all products for neglected diseases. This experience will help us a great deal in negotiating new contracts with

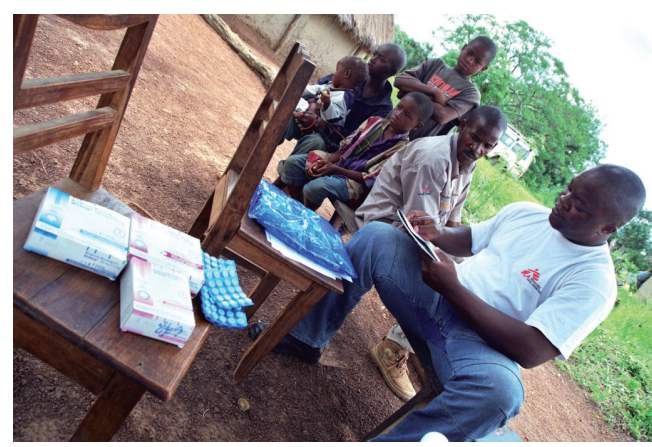

ASAQ in use by Médecins sans Frontières in Guinea. other companies, as we can use our experiences with Sanofi Aventis as a model for future discussions. I feel that at both governmental and local levels there is now a consensus that IP will not stimulate R\&D for neglected diseases.

Some feel that there exist other, more significant barriers to treatment access than patents \& patent law. What are your thoughts on this?

The relationship between IP and high costs is a relatively easy one to draw. When you have a monopolistic situation, you can raise prices. So competition is the best way to reduce costs. 
I think regulatory requirements are also extremely important, and are another major obstacle to $\mathrm{R} \& \mathrm{D}$, as is the availability of products. Of course, taxes on a country level are also significant, but I think this is a different issue, since you cannot tackle a major public health problem without government commitment.

What are your opinions on GlaxoSmithKline's (GSK's) announcement that they intend to decrease the cost of medicines for the poorest countries \& develop 'patent pools' to share knowledge about potential drugs that are currently protected by patents?

I think it is a positive move because it confirms two things. Firstly, it shows that GSK acknowledge they accept the concept of differential pricing, even if, in my opinion, it is only one of numerous solutions. I feel that this situation is too complex to be dealt with using only one approach. Second, their acceptance of the idea of a 'patent pool' is confirmation that for neglected diseases IP is not a key issue.

Q A significant issue facing neglected disease drug development concerns the regulatory frameworks in disease-endemic countries. What are your thoughts on overcoming this challenge?

For the time being, we are completely reliant on national regulatory authorities. There is no possibility of the FDA being the regulatory body for the rest of the world. That is not the solution, nor is it acceptable to have a single group from a single country deciding the risk-benefit for another population. It is crucial to involve regulatory authorities in disease-endemic countries, both in terms of R\&D and of access to drugs. At the same time, however, we know that some regulatory authorities are weak and not able to carry out this role.

This is why - and I refer to the lessons we have learned through ASAQ and ASMQ, for example - we are trying to combine the involvement of regulatory authorities in Brazil, Africa or India with the WHO prequalification program, an international system that draws on the expertise of many different countries. Therefore, an international body working with national authorities is one model that could be used in the future for neglected diseases.

\section{Past \& future perspectives}

What, in your opinion, have been the most significant developments in neglected disease $R \& D$ since you began your career?

This is difficult question! I am a medical doctor, and I began my career working for Médecins Sans Frontières, treating people with very difficult conditions. While in Uganda, I was confronted with the use of melarsoprol, an arsenic derivate, to treat patients suffering from sleeping sickness, while knowing that it could kill one in 20 patients who received it.

The big change that has occurred is that today we have the ability to use the knowledge we have for patients better. At the DNDi we are trying to make the dream of..... possible. Hopefully we will not be unique in this regard, and our model will be used for many other therapeutic areas, and help change the lives of many people.

\section{Since you began your career, how has the attention} given to neglected diseases changed?

What has changed is the awareness of the problem. For example, neglected diseases have been discussed several times at very high levels, for example the G8 meeting and by the UN. So what was once discussed between a few people 25 years ago is now being discussed by high-level decision makers. In this sense there has been a big change.

\section{DNDi's main accomplishments up to 2009}

- Largest ever R\&D portfolio of potential new treatments for sleeping sickness, visceral leishmaniasis and Chagas disease.

- Clinical research capacity established in extremely difficult, resource-poor, rural settings in Africa.

- Two fixed-dose antimalarials (ASAQ and ASMQ) delivered to patients in Africa and Latin America, respectively:

- The first pediatric strengths in such a fixeddose combination.

- Delivery of mllions of these child-friendly, easy-to-use products.

- Improved sleeping sickness treatments:

- NECT, a simpler, safer, shorter treatment for stage 2 sleeping sickness, has successfully completed Phase III clinical trials.

- Fexinidazole, a new sleeping sickness drug candidate, begins clinical trials in 2009. 




Are you optimistic about what the short- to medium-term future holds?

Not yet, because I think the successes are still few and the gaps are still big. We do not have a good perspective for improving treatments for Chagas and other diseases for example, so there are still many challenges.

What words of encouragement would you give young scientists who might be envisioning a career in neglected disease research?

I think I would reiterate this point: today it is possible for a young scientist to harness a great deal of creativity and new knowledge for the development of new drugs, vaccines, and technologies for those living in very difficult conditions.

\section{Biography}

Dr Bernard Pécoul has been the Executive Director of DNDi since October 2003 and played a key role in its formation while at the Access to Essential Medicines Campaign of Médecins Sans Frontières (MSF). Prior to that, he was Executive Director of MSF-France, co-founder of Epicentre and a MSF field physician across Africa, Latin America and Asia. He obtained his Medical Docterate from the University of Clermont Ferrand, France, and his Masters in Public Health from Tulane University, USA. 